\title{
Fabrication of sharp silicon arrays to wound Caenorhabditis elegans
}

\begin{abstract}
Jérôme Belougne ${ }^{1}$, Igor Ozerov ${ }^{2 *}$, Céline Caillard ${ }^{1}$, Frédéric Bedu² \& Jonathan J. Ewbank ${ }^{1 *}$
Understanding how animals respond to injury and how wounds heal remains a challenge. These questions can be addressed using genetically tractable animals, including the nematode Caenorhabditis elegans. Given its small size, the current methods for inflicting wounds in a controlled manner are demanding. To facilitate and accelerate the procedure, we fabricated regular arrays of pyramidal features ("pins") sharp enough to pierce the tough nematode cuticle. The pyramids were made from monocrystalline silicon wafers that were micro-structured using optical lithography and alkaline wet etching. The fabrication protocol and the geometry of the pins, determined by electron microscopy, are described in detail. We also used electron microscopy to characterize the different types of injury caused by these pins. Upon wounding, C. elegans expresses genes encoding antimicrobial peptides. A comparison of the induction of antimicrobial peptide gene expression using traditional needles and the pin arrays demonstrates the utility of this new method.
\end{abstract}

As part of the investigation of innate immune defences in C. elegans, worms are generally wounded in one of two ways. For precise cell-biological studies, individual worms mounted on glass slides can be injured using a laser coupled with an inverted microscope. As a complementary method, worms can be wounded manually, using standard glass microinjection needles ${ }^{1}$. Both techniques are time-consuming and require meticulousness. More than 20 years ago, a method for genetically transforming nematodes using arrays of silicon micromechanical piercing structures was published ${ }^{2,3}$. This inspired us to investigate the possible use of such silicon arrays as a tool to wound C. elegans in a relatively uniform but less painstaking manner.

Silicon is the most widely used material in the microelectronics industry and in the production of micro-electromechanical systems. It is abundant, inexpensive and mechanically robust ${ }^{4}$. High-quality silicon wafers are available commercially and fabrication processes for silicon features have been developed for more than 50 years ${ }^{5}$. Silicon microstructures have been already successfully used for piercing of biological objects ${ }^{2}$ and for the fabrication of microneedles ${ }^{6}$ for gene and drug delivery ${ }^{7}$. These studies have shown that silicon is a chemically and mechanically stable biocompatible material. Despite the apparent simplicity of the method described by Trimmer and colleagues ${ }^{2}$, there appears to be no other study using these arrays to transform any nematode species. On the other hand, such arrays could be well-suited for use as tools to wound worms ${ }^{8}$. Unfortunately, neither the arrays nor a protocol for their fabrication are available any more (W. Trimmer, personal communication). In order to fabricate equivalent microstructures, we therefore developed our own protocol, based on optical lithography and alkaline wet etching.

\section{Materials and Methods}

Array production. As initial material, we used n-type monocrystalline silicon wafers doped by phosphorus $(5-10 \Omega \mathrm{cm})$ oriented with [100] as the surface plane (from Sil'tronix, France). The wafers were polished on both sides and covered with a $290 \mathrm{~nm}$-thick thermally grown oxide layer. We used two sizes of wafers with diameters of 1 inch $(25.4 \pm 0.3 \mathrm{~mm})$ and 4 inches $(100 \pm 0.3 \mathrm{~mm})$.

All the microfabrication processes were carried out in a clean room of air purity class ISO 6 . The wafers were cleaned in successive baths of acetone and isopropyl alcohol (IPA) with ultrasonic agitation, then rinsed with deionized water and dried under a clean nitrogen flow. In order to improve the adhesion of the photoresist and to remove the traces of organic solvents and humidity, the wafers were exposed to oxygen plasma at a temperature of $150^{\circ} \mathrm{C}$ for 10 minutes in a plasma chamber (Nanoplas, France). The radiofrequency power for plasma excitation was 200W. Then, the wafers were spin-coated with a positive photoresist (Microposit S1813 from MicroChem) and exposed to UV-light using MJB-4 (Süss MicroTec, Germany) or PLA-501 (Canon, Japan) optical lithography tools through a mask of round features.

\footnotetext{
${ }^{1}$ Aix Marseille Univ, CNRS, INSERM, CIML, Turing Centre for Living Systems, Marseille, France. ${ }^{2}$ Aix Marseille Univ, CNRS, CINaM, Marseille, France. *email: ozerov@cinam.univ-mrs.fr; ewbank@ciml.univ-mrs.fr
} 


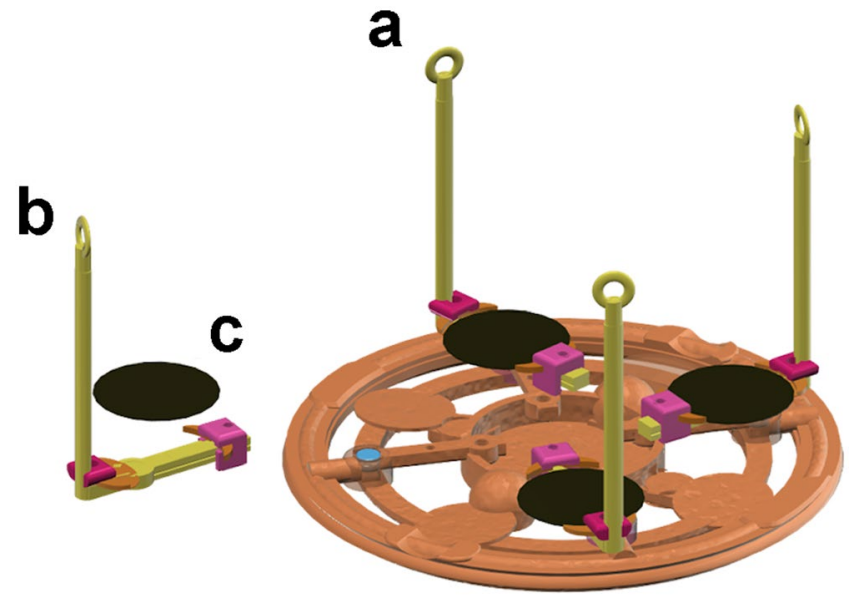

Figure 1. Drawing of a sample holder used for homogeneous $\mathrm{KOH}$ etching of multiple one-inch wafers (Autodesk 123 Design software). (a) Main support with magnets; (b) Arms for easy removal from etching solution; (c) One-inch $(2.54 \mathrm{~cm})$ diameter wafer to be etched.

We studied two types of arrays using masks with different geometries: hexagonal and square arrays. The hexagonal arrays were denser than the square ones, but in both cases we kept the distance between the features large enough in order to prevent possible proximity effects. During the lithography step, we usually oriented the $\mathrm{x}$-axis of the arrays along the [110] silicon crystalline axis in order to cut the samples in between the features during the silicon cleavage; the orientation should not affect the final shape of individual microfeatures 9 .

The feature diameters were 75,100 and $300 \mu \mathrm{m}$ and the center-to-center distances were 100, 133 and 400 $\mu m$ respectively in the hexagonal arrays. The mask designs for the 75 and $100 \mu m$ feature arrays are shown in Supplementary Fig. S1. For square arrays, we used only $300 \mu m$ features with 400 and $600 \mu m$ center-to-center distances (pitch). After UV light exposure the features were developed in a commercially available solution (Microposit MF-319) containing tetramethylammonium hydroxide (TMAH). The thickness of the deposited photoresist was about $1.4 \mu \mathrm{m}$ and it was monitored by a mechanical contact profilometer (Dektak, Bruker).

The features were transferred from the resist layer to silicon dioxide by reactive ion etching (MG-200, Plassys, France). The silicon dioxide was completely removed from the topside of the wafers in the areas between the features that were protected by photoresist. We used $\mathrm{CHF}_{3}$ reactive plasma because of its good selectivity in etching of $\mathrm{SiO}_{2}$, silicon and the photoresist polymer ${ }^{10}$. The remaining photoresist was removed in a solution of 1165 (from Microposit) containing N-methyl-2-pyrrolidone. We confirmed complete removal of the oxide layer in the areas between the round features by contact profilometry. Finally, several wafers were directly processed by alkaline solution while the other wafers were cut in $1 \mathrm{~cm} \mathrm{x} 1 \mathrm{~cm}$ square pieces along [110] the crystalline directions of the silicon crystal using a diamond scriber.

Each sample was exposed to potassium hydroxide $(\mathrm{KOH})$ solution in order to etch silicon in the areas not protected by the $\mathrm{SiO}_{2}$ hard mask. Wet chemical attack by hydroxide solutions is known to be anisotropic because $\mathrm{KOH}$ etches different crystalline planes with significantly different rates ${ }^{11}$. We chose a relatively high $\mathrm{KOH}$ concentration of $45 \%$ (in deionized water; Fluka) because the resulting surface quality is known to be better for highly concentrated solutions (see page 40 in Fruhauf et al. ${ }^{5}$ ). In several experimental series, we also saturated the solution by addition of isopropanol (IPA) or ethanol.

A borosilicate glass beaker containing the etching solution was placed into a water bath on a regulated hotplate. The etching solution was kept at a fixed temperature during the etching in order to control the shape of the obtained features, which was also influenced by the etching time. The temperature was measured by a thermometer immersed in the $\mathrm{KOH}$ solution. We developed a series of custom designed magnetic 3-D printed sample holders allowing constant uniform agitation for the samples of different sizes and shapes ${ }^{12}$. The samples were fixed on the magnetic holders fabricated from a temperature resistant photopolymer plastic resin (Formlabs High Temp Resin) and then immersed in the etching solution. Before their first use, the sample holders were rinsed in a hot $\mathrm{KOH}$ solution for at least one hour, to remove polymer residues that otherwise interfered with alkaline etching. The samples were agitated at high speed in hot alkaline solution on a standard bench-top magnetic stirrer in order to homogenize the etching and to disperse hydrogen bubbles that appear during the chemical reaction of silicon with hydroxide ions ${ }^{11}$. It was possible to install up to four small samples onto the holders (Fig. 1) and to process them in the same etching run. The samples could be then extracted one by one after the desired etching times. The 4 inch wafers were processed one-by-one. In order to keep the most stable conditions, we used the same quantity of $\mathrm{KOH}(650-750 \mathrm{ml})$ in the beaker and we changed the solution regularly to prevent the alteration of its composition through the introduction of chemical reaction products and by evaporation.

The typical etching time in $\mathrm{KOH}$ solution depended on the feature size. For the $75 \mu \mathrm{m}$ feature arrays it was between 50-60 minutes and for $300 \mu \mathrm{m}$ feature arrays, about 90 minutes at $65^{\circ} \mathrm{C}$. After the etching, the samples were rinsed in deionized water in order to stop the reaction, then dried under clean nitrogen and observed by optical and scanning electron microscopy (SEM). The optical microscopy gives an immediate evaluation of the etching homogeneity and microscopic defects present on the sample surface. Moreover, the feature base size, the 

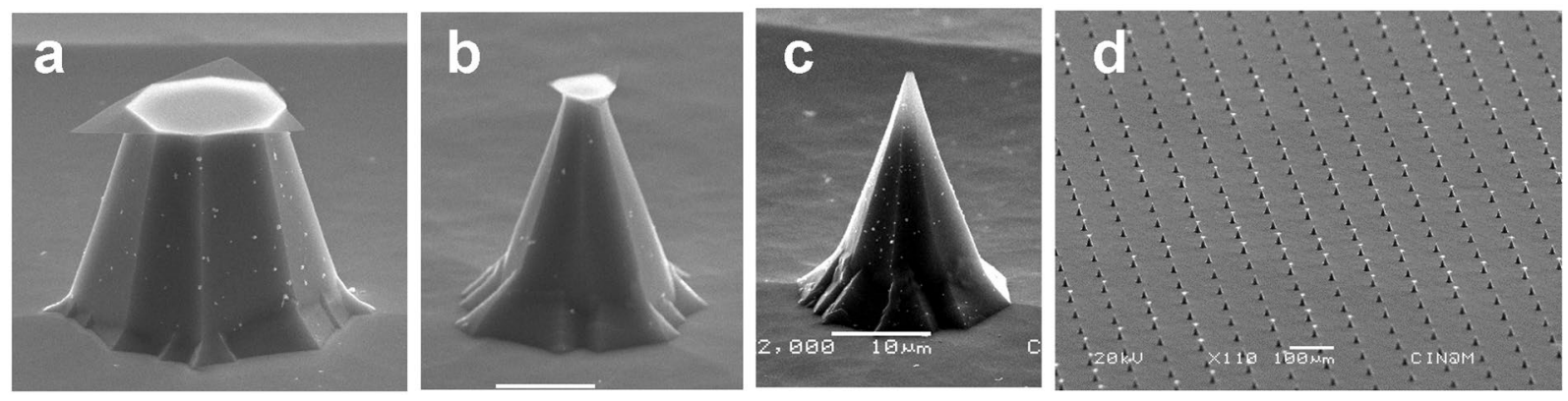

Figure 2. Silicon pyramids in a $75 \mu \mathrm{m}$ array etched in fresh $45 \% \mathrm{KOH}$ solution at $\mathrm{T}=63^{\circ} \mathrm{C}$, sample holder rotation speed 170 r.p.m for 100 minutes (a), 110 minutes (b), 114 minutes, followed by an additional 4 minutes, after removal and inspection with a stereomicroscope (c). The 3 images are at the same magnification. (d) Large array of homogeneous pins at the end of the etching period. Of the 342 pins in this electron photomicrograph, 130 retain the "hat" that is subsequently removed in a pure water ultrasound bath. Manufacturing parameters: $75 \mu \mathrm{m}$ array; $750 \mathrm{ml}$ fresh $45 \% \mathrm{KOH} ; \mathrm{T}=72^{\circ} \mathrm{C} ; 100 \mathrm{rpm}$. All the samples were tilted in the SEM at $70^{\circ}$.

undercut depth and the presence of any remaining $\mathrm{SiO}_{2}$ mask can also be estimated from optical microscopy. In order to measure the size and characterize the shape of pyramids, however, we needed to use SEM and to tilt the samples from normal incidence to angles from 40 to $70^{\circ}$. For SEM observation we used a tungsten-filament JEOL JSM-5910 microscope with a typical electron acceleration voltage of $20-30 \mathrm{kV}$.

After the observations, samples could be returned to the $\mathrm{KOH}$ solution in order to continue the etching to obtain the desired pin shape of the features. To avoid the risk of over-etching, samples that were almost finished could be placed into a ultrasonic bath (LEO-801 Bioblock Scientific, Illkirch, France) for 5-10 minutes in order to obtain sharp features by mechanical breaking of the remaining silicon at the top of the pin tip. Removal was effective provided that the junction between the two parts was of the order of $2 \mu \mathrm{m}$ thick. Wider junctions required longer periods of ultrasound treatment to break and this was associated with a fracturing of the apex of the pyramidal structure.

Worm methods. The strains IG274 ${ }^{1}$ and IG1061 13 carrying the frIs7 ( $n l p-29 p:: G F P$; col-12p::DsRed2) integrated array in the wild-type or sta-2(ok1860) background, respectively, were cultured under standard conditions, i.e. on E. coli OP50 on NGM agar plates ${ }^{14}$. Wounding with a micro-injection needle was performed as previously described $^{15}$. For experiments using the silicon arrays, the wafers were mounted either on plastic handles using a cyanoacrylate adhesive ("superglue") or on the end of syringe plungers, using household double-sided adhesive tape. Synchronized populations of young adult worms, obtained by alkaline hypochlorite treatment ${ }^{14}$, were transferred to plates without bacteria, rapidly wounded by bringing the wafer down with gentle pressure, repeatedly in a tiling fashion to cover the surface of the plate before transfer to a fresh OP50-seeded plate. In both cases, upregulation of $n l p-29 p:: G F P$ reporter gene levels were quantified after 6 hours with the COPAS Biosort (Union Biometrica $)^{15}$. Adult worms were stained with Hoechst 33258 as previously described ${ }^{16}$, and with DAPI $(2.5 \mu g /$ $\mathrm{ml}$ in $0.5 \mathrm{mM}$ levamisole) for 15 minutes at room temperature, with agitation, followed by 3 washes with $50 \mathrm{mM}$ $\mathrm{NaCl}$.

Electron microscopy. Worms were fixed in $2 \%$ glutaraldehyde in $0.12 \mathrm{M}$ phosphate buffer overnight. They were postfixed in $1 \%$ osmium in distilled water for 30 minutes. Dehydration in a graded ethanol series preceded drying from liquid carbon dioxide by the critical point method. The worms were then gently transferred to a carbon-taped stub for gold sputtering (10' at $15 \mathrm{~mA}$, Edwards). Imaging was carried out on an FEI Teneo VS running in high vacuum mode, at $5 \mathrm{kV}$ and using an ETD secondary electron detector.

\section{Results}

Array microfabrication. In order to obtain an initial estimate of the required etching time and the final shape of the pins, we modelled the etching process using ACES software ${ }^{17}$, taking the data from Seidel et al. ${ }^{18}$. We simulated different mask shapes including circles and squares (see Supplementary Fig. S2) and we chose the former in order to simplify process optimization and to get closest to the desired final pin shape. The round features with initial mask diameters of 75, 100 and $300 \mu \mathrm{m}$ were organized in arrays with separation distances sufficient for pyramid pin development. The pin density, i.e. the center-to-center pin distance, was chosen to be small enough to wound young adult $C$. elegans that are typically about $1 \mathrm{~mm}$ in length.

When wafers were removed at intervals from the $\mathrm{KOH}$ solution, the successive steps of etching for typical individual silicon pyramids, using a hexagonal mask with the pitch of $100 \mu \mathrm{m}$ and initial feature diameter of $75 \mu \mathrm{m}$, could be followed using SEM (Fig. $2 \mathrm{a}-\mathrm{c}$ ). This was straightforward as they required no preparation or treatment before imaging. After 100 minutes of etching, one can clearly see the pyramid with an octagonal trunk, resulting from undercutting of silicon under the $\mathrm{SiO}_{2}$ mask, with a lateral size of about $30 \mu \mathrm{m}$, corresponding to the pyramid base, and $16 \mu \mathrm{m}$ corresponding to the pyramid top. The remaining $\mathrm{SiO}_{2}$ mask is clearly visible on the top of the pyramid (Fig. 2a). After 110 minutes of alkaline etching, one can see that the under-etching is deeper and the lateral sizes of both base and top of the pyramid are decreased to 25 and $5.5 \mu \mathrm{m}$, respectively (Fig. 2b). Finally, after etching in the same solution for a total time of 118 minutes, the remaining pyramid has a base size of 
a

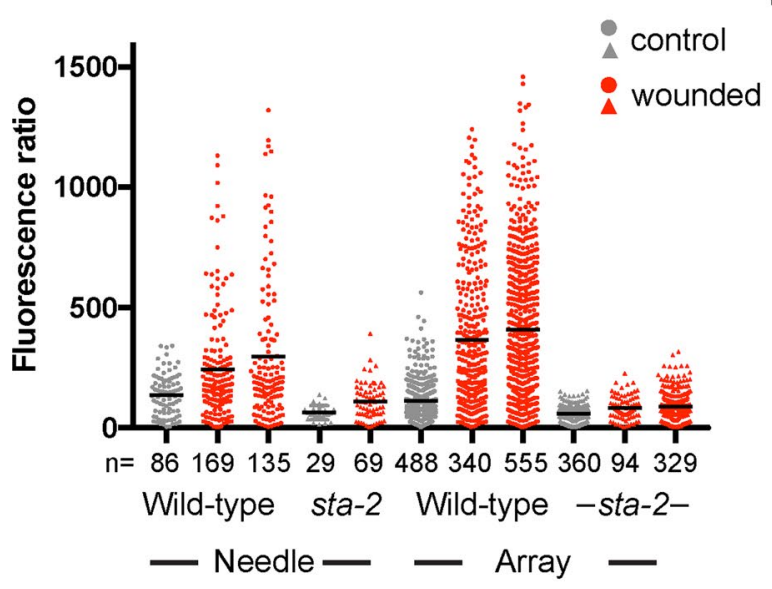

b

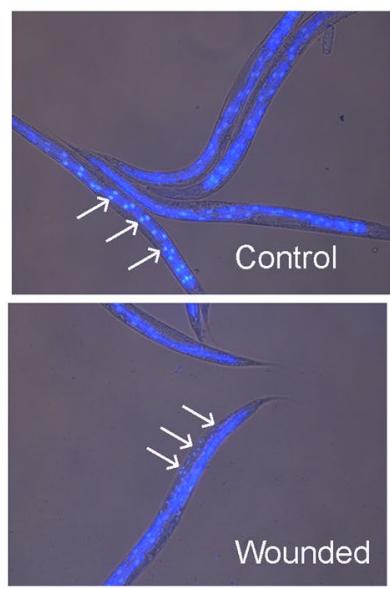

Figure 3. Efficient wounding of C. elegans with a silicon array. (a) Quantification of the change of reporter gene expression provoked by wounding with a needle (5 left-hand columns), or arrays, worms carrying frIs 7 in the wild-type or sta-2 mutant background (circles and triangles, respectively). The fluorescence ratio (Green/TOF in arbitrary but constant units) is shown for the indicated number of worms for each condition (n). The bars indicate the means. (b) Representative images of control (top) and wounded (bottom) adult wild-type worms stained with Hoechst 33258. In the control animals, only staining of the intestinal nuclei is apparent (arrows), while in the array-wounded worms nuclei in the epidermis (arrows) are also stained.

$21 \mu \mathrm{m}$ and a very sharp tip. This form most closely resembles the pins previously described ${ }^{2}$ (see Supplementary Fig. S3). Overall, the selectivity in the etching of $\mathrm{Si}$ and $\mathrm{SiO}_{2}$ was very high, and the oxide mask protected the silicon from alkaline attack relatively well (Fig. 2a-c). KOH solution attacks (100) Si planes and, because of the strong undercut, the thin $290 \mathrm{~nm}$-thick $\mathrm{SiO}_{2}$ layer remained only in an area with a size of 5-7 $\mu \mathrm{m}$. For some pyramids, we observed complete removal of the $\mathrm{SiO}_{2}$ protection, but in general, the etching was very homogeneous when we used our custom-designed 3D-printed rotating sample holder (Fig. 1) at speeds of 100 to $180 \mathrm{rpm}$. In earlier work, the crystalline planes that form the pyramid pins were identified as $(411)^{3,6,19}$. These crystal planes give a tetragonal tip similar to those observed for Si etching in Triton X-100 supplemented TMAH ${ }^{20}$. In our case, the tips of the pins are octagonal with the shapes similar to those reported by Wilke et al. ${ }^{9,21}$. Those authors identified the crystal planes forming the main sharp part of pyramids as belonging to (312) family, while the crystalline planes of the base were identified as $(228)^{21}$. In order to ensure scalability of the process, we compared the shape of the pyramids obtained using 75 and $100 \mu \mathrm{m}$ masks and found that the angles of the tips were similar; only the pin base size and height changed (see Supplementary Fig. S4). On the other hand, the shape of the tips was dependent on the etching bath conditions, in particular on the freshness of the $\mathrm{KOH}$ solution. Aging of the solution favored the formation of flat pyramid bases, consistent with previous observations ${ }^{21}$. The addition of ethanol, Triton X-100 or IPA also favored the formation of pyramids without sharp tips. The addition of IPA, for example, stabilized the etching process. Thus pyramid surfaces were very smooth without defects, but pyramids were formed by only their bases (see Supplementary Fig. S5). In this case also, the crystal planes forming the pyramids correspond to those previously described ${ }^{21}$. On the basis of these results, for subsequent etchings, we used baths of fresh aqueous $\mathrm{KOH}$ solution, without additives, for each series of samples to obtain the optimal shape of the pyramidal pins and to diminish the base development. We also increased the etching temperature because higher temperatures allowed us to decrease the etching time which was otherwise inconveniently long for the arrays with initial sizes of 100 and $300 \mu \mathrm{m}$. For $75 \mu \mathrm{m}$ arrays the typical etching time was about 45 minutes for standard etching temperatures of $70-72^{\circ}$ to obtain sharp tips (see Supplementary Fig. S6a-c). Once the $\mathrm{SiO}_{2}$ protection was gone, sharp pyramids were rapidly attacked by $\mathrm{KOH}$. Their height as well as width diminished and when over-etched, only the more resistant crystalline planes close to the base remained (see Supplementary Fig. S6d). This instability made the etching process very time-sensitive. We therefore etched samples in $\mathrm{KOH}$ for a time slightly inferior to that expected for complete $\mathrm{SiO}_{2}$ removal, then examined the features using SEM. If necessary we then returned the sample to the $\mathrm{KOH}$ solution to finish the process. Because of the consumption of all the silicon under the $\mathrm{SiO}_{2}$ mask, the protective "hat" part was readily removed when the wafer was rinsed under a flow of deionized water at the end of the etching process. When the pyramid tip size approached $2-5 \mu \mathrm{m}$, it was also possible to finish the fabrication by mechanical breaking of the remaining "hat" in a pure water ultrasound bath. In both cases, the remaining pyramids had very sharp tips. Although requiring precision, by standardising the different steps of the procedure, we were able to make large arrays of regularly structured pyramidal pins (Fig. 2d) in a routine and reproducible manner.

Efficient wounding of $C$. elegans. To make handling of the arrays easier, they were adhered to a short plastic rod or on the end of syringe plungers. To wound young adult C. elegans, an array was brought down gently onto a synchronised population of worms on an agar plate. The array was lifted, moved and brought down again. The light pattern of indentations left on the surface of the agar was used to ensure complete coverage of 
a

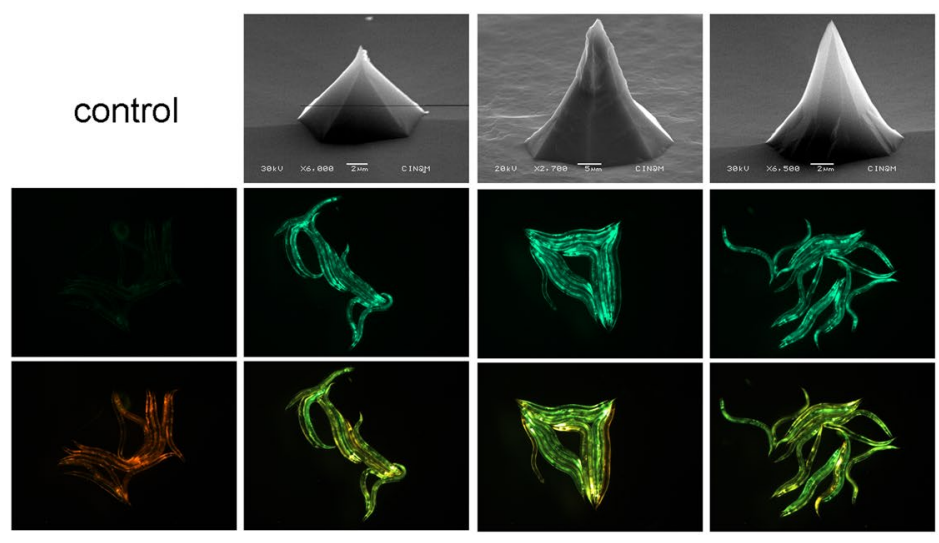

b

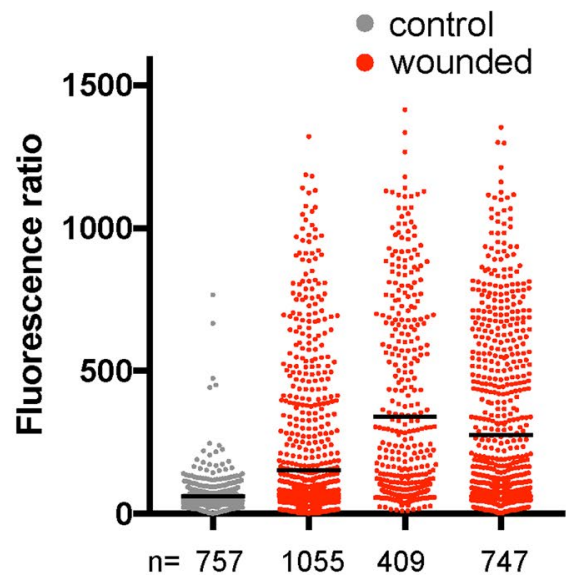

Figure 4. Wounding of $C$. elegans with different silicon arrays. (a) Top row shows SEM images of 3 different arrays (left to right: H75-42, H100-9, H75-46). Note that, as indicated, the magnification is not the same for all 3, hence the scale bars are different too. The other 2 rows are GFP (middle row) or mixed GFP/dsRed fluorescence signals for representative groups of worms carrying frIs 7 picked because they appeared to have a normal morphology (i.e. were not badly wounded) after treatment with the indicated array, compared to nonwounded controls (left-hand column). (b) Quantification of the change of reporter gene expression provoked by wounding with the 3 arrays, in the same order as in a, in worms carrying frIs7. Details are as in the previous figure.
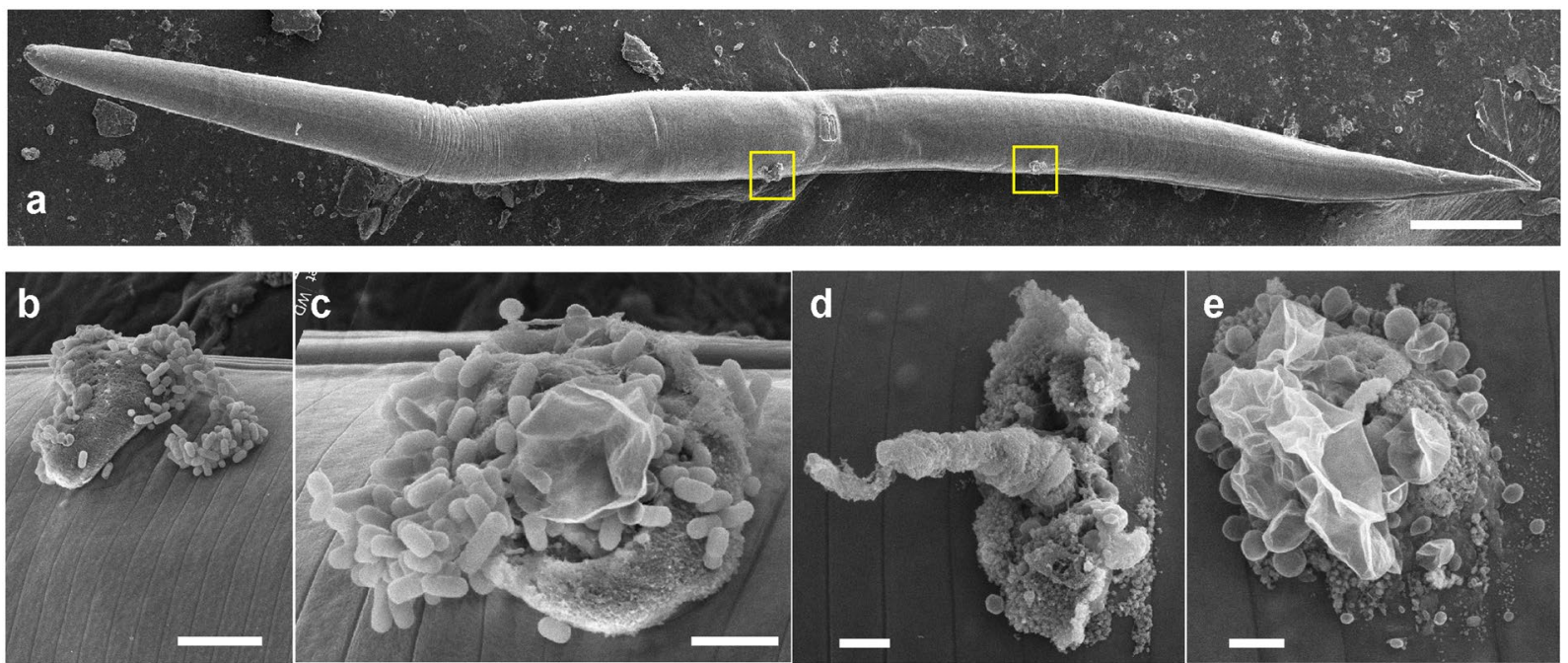

Figure 5. Wounds inflicted on C. elegans by silicon arrays. (a) SEM images of an adult hermaphrodite worm that showed normal movement and overall morphology and had strong $n l p-29 p:: G F P$ reporter gene expression 6 hours after wounding. The worm has at least 2 lesions (boxed in yellow). These are shown at higher magnification in (b,c). In common with many lesions, the extruded material was found covered in bacteria. Two further wounds from a different worm are also shown (d,e). Scale bars, 100, 5, 2, 1, 1 micron, (a-e).

the population. Signs of wounding were visible through a dissecting microscope. These ranged from the type of small hernia-like extrusions often seen with needle-wounds, through more extensive loss of internal content (see Supplementary Fig. S7), to immobility and complete loss of normal body structure. In a typical experiment, more than two thirds $(67.3 \%)$ of the worms $(n=584)$ were fully motile 4 hours after wounding. One of the consequences of wounding or fungal infection of the C. elegans epidermis is the rapid induction of antimicrobial peptide (AMP) gene expression. This can be monitored through the use of transgenic worms carrying fluorescent reporter genes. The most extensively characterised strain, IG274, carries the integrated transgene frIs 7 , with 2 reporter genes, dsRed and GFP under the control of a constitutive epidermal promoter, col-12, and the infection-inducible $n l p-29$ promoter, respectively. In the absence of pathogens or injury, IG274 worms appear red, but express GFP strongly when wounded or infected. These changes can be monitored visually or quantified using the COPAS Biosort ${ }^{1}$. We compared the induction of $n l p-29 p:: G F P$ provoked by wounding by hand with a 


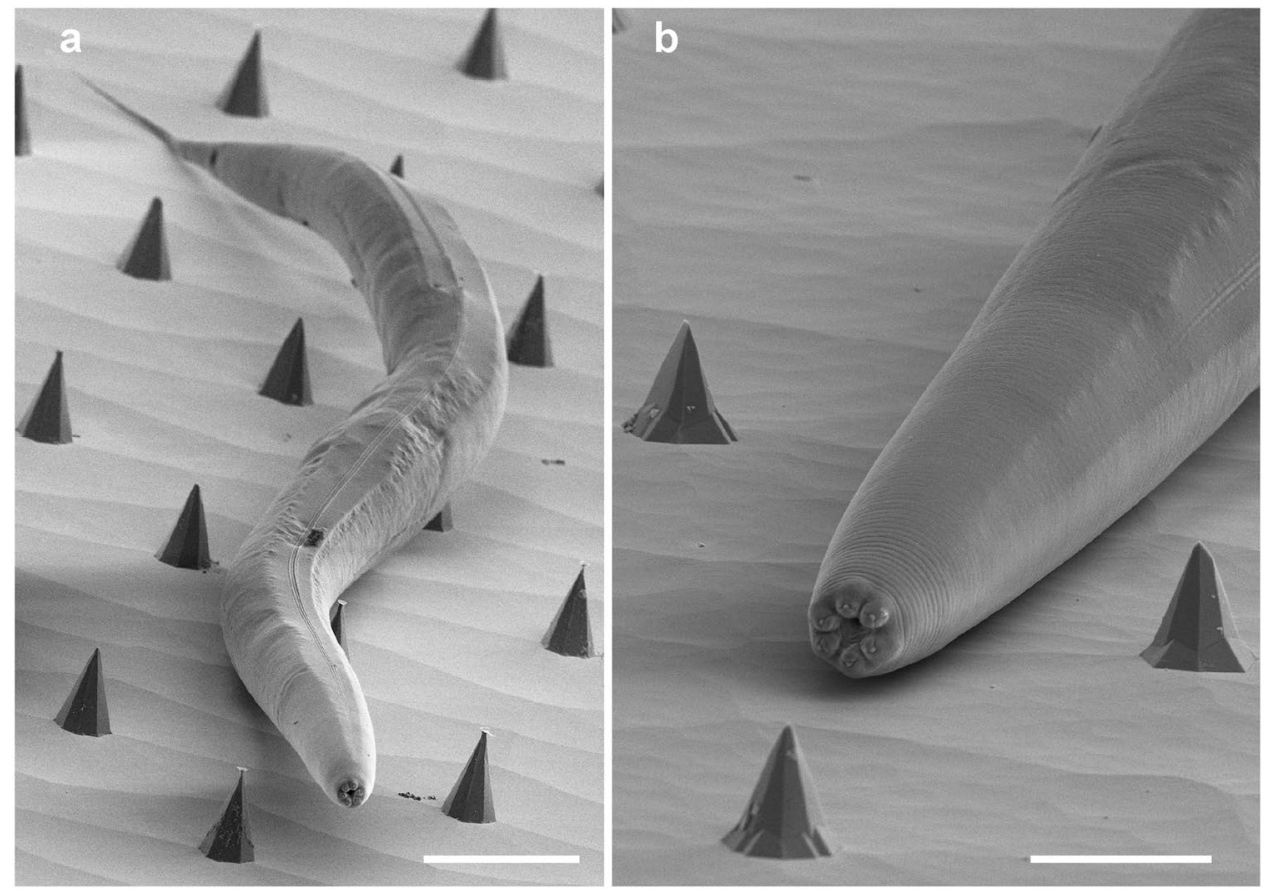

Figure 6. Artificial montage to illustrate the relative sizes of C. elegans and pins, with adult hermaphrodite worms fixed for EM placed directly on silicon pyramids in $100 \mu \mathrm{m}$ (a) and $75 \mu \mathrm{m}$ (b) arrays. Scale bars, 50 and 20 micron, (a,b).

glass needle or with the silicon pin arrays. As expected, manual wounding with a microinjection needle caused a substantial increase in average GFP expression, and this was almost completely abrogated in a sta-2 mutant, consistent with previous observations ${ }^{13}$. There was an even more marked increase when worms, whether at a lower or higher density on the culture plate, were wounded with a silicon array. Again, this response was dependent upon sta-2 (Fig. 3a), consistent with both methods activating AMP gene expression by the same canonical signal transduction pathway. Importantly, in addition to producing equivalent results as the standard needle method, the use of the array simplified and accelerated the procedure substantially. An experienced researcher with good manual dexterity can reasonably wound one worm every $2-4$ seconds. With the arrays, no training was required and the speed of wounding was increased 10-50 fold, as hundreds of worms could be wounded in less than 30 seconds. Consistent with the fact that the arrays pierced the collagen-rich cuticle that renders C. elegans impermeable to most compounds $\mathrm{s}^{22}$, we observed extensive staining of epidermal nuclei with dyes that under normal culture conditions, in non-molting animals, only stain the nuclei of intestinal cells ${ }^{23}$ (Fig. 3b). In severely wounded worms, germline staining was often apparent. Among worms without marked tissue extrusion, in only a very small proportion (3.6\%, $\mathrm{n}=83$ ) was germline nuclear staining observed (see Supplementary Fig. S8). Having established the potential utility of the arrays, we went on to evaluate the efficiency of wounding using pins of different sizes, spacing and geometries. While the average fluorescence ratio was greater when the pins were longer, the geometry did not appear to be critical (Fig. 4). We found that we could use the arrays multiple times. Even after repeated use, we observed little abrasion of the pin apex (see Supplementary Fig. S9). When 6 hours after wounding, we examined normally moving worms that strongly expressed the $n l p-29 p:: G F P$ reporter, with rare exceptions, these worms displayed only minor tissue damage, as judged by light microscopy (see Supplementary Fig. S10). Through SEM, we characterised in more detail some of these animals. We observed a range of lesions, typically of the order of $5 \mu \mathrm{m}$ in size. In all cases $(\mathrm{n}>20)$, it appeared that material had been extruded from inside the worm. In addition to amorphous extrusions, there were often diaphanous folds (Fig. 5; see Supplementary Fig. S11), which we take to be damaged cuticle.

\section{Discussion}

Using an alkaline etching technique, we produced arrays of silicon pyramidal pins. Contrary to the fabrication of concave features on $\mathrm{Si} \mathrm{(100)} \mathrm{which} \mathrm{is} \mathrm{usually} \mathrm{self-limiting} \mathrm{when} \mathrm{the} \mathrm{slowly} \mathrm{etched} \mathrm{(111)} \mathrm{surfaces} \mathrm{are} \mathrm{reached,}$ the fabrication of convex features on $\mathrm{Si}(100)$ by alkaline etching is more challenging ${ }^{5}$. Two processes occur at the same time: etching of the (100) crystalline surface directed towards the wafer depth in the areas that are not protected by a mask and undercutting of silicon features in a lateral direction under the mask despite the protection. The final shape of the obtained features depends on the difference in the rates of these two processes $^{24}$. For example, the etching rate for (111) silicon crystalline planes is more than 100 times slower than for the other planes such as $(100)^{25,26}$, and it is possible to obtain sharp features with a pyramidal shape both for (100) and (111) oriented wafers ${ }^{20}$. Numerous models have been proposed to explain the convex corner undercutting $^{25}$ and its compensation ${ }^{26}$ and, more generally, anisotropic etching of silicon ${ }^{27}$. The main complexity in the description of experimental procedures arises from the fact that multiple parameters have been reported to 
affect the undercutting rate: the solution temperature ${ }^{5}, \mathrm{KOH}$ or TMAH concentration, freshness of the alkaline solution ${ }^{21}$ as well as the introduction of different chemical additives to the solutions such as $\mathrm{I}_{2} / \mathrm{KI}^{28}$, the surfactant Triton-X-100 20,29 or $\mathrm{IPA}^{5}$. In our fabrication process, we used only fresh aqueous $\mathrm{KOH}$ solution baths for each sample series and we optimized the shape of the pyramidal pins to be suitable for biological experiments. Only etching in pure $\mathrm{KOH}$ solutions allowed us to obtain sharp pyramids. Addition of ethyl or isopropyl alcohol or Triton to aqueous $\mathrm{KOH}$ solutions favoured the formation of crystal planes forming flat pyramids. We developed a series of sample holders for the wafers of different sizes from $1 \mathrm{~cm}^{2}$ up to $10 \mathrm{~cm}$ in diameter, resistant to $\mathrm{KOH}$ and alcohol solutions up to the temperatures of more than $80^{\circ} \mathrm{C}$ necessary to ensure homogeneous etching over large surfaces. Very sharp pyramidal needles were organized into arrays with a pitch of 100-133 $\mu \mathrm{m}$, dense enough to wound young adult C. elegans hermaphrodites that are around $1 \mathrm{~mm}$ in length (Fig. 6).

In the original publication, piercing structures similar to the ones described here were claimed to have been used to transform the entomopathogenic nematode Heterorhabditis bacteriophora in an extremely rapid and efficient manner ${ }^{2}$. Despite the obvious attraction for such a simple method, no other publication has ever reported the successful replication of this work. Parasitic nematodes are notoriously difficult to transform, compared to $C$. elegans for which micro-injection-based transformation is a standard laboratory technique. In our hands, we were unable to obtain any evidence for transformation of C. elegans using our silicon pin arrays. Neither more than 30 trials replicating the conditions used previously ${ }^{2}$, nor more than 150 trials combining array-mediated wounding followed by electroporation using hygromycin resistance as a method of selection yielded any transgenic worms (JB and CC, unpublished; full details available upon request). Thus, unfortunately, it appears that their utility for making transgenic nematodes is limited at best. On the other hand, these arrays enormously accelerate and simplify the generation of large populations of wounded C. elegans. This opens up the potential for generating samples in a sufficient volume to allow transciptomic, proteomic or metabolomic analyses of the response to wounding, further strengthening the utility of C. elegans in this domain ${ }^{30,31}$.

Received: 19 September 2019; Accepted: 2 January 2020;

Published online: 27 February 2020

\section{References}

1. Pujol, N. et al. Distinct innate immune responses to infection and wounding in the C. elegans epidermis. Curr. Biol. 18, 481-9, https://doi.org/10.1016/j.cub.2008.02.079 (2008).

2. Hashmi, S. et al. Genetic transformation of nematodes using arrays of micromechanical piercing structures. Bio. Techniques 19, 766-770 (1995).

3. Trimmer, W. et al. Injection of DNA into plant and animal tissues with micromechanical piercing structures.In Proceedings IEEE Micro Electro Mechanical Systems. 1995, 111, https://doi.org/10.1109/MEMSYS.1995.472544 (IEEE, Amsterdam, Netherlands, 1995).

4. Hopcroft, M. A., Nix, W. D. \& Kenny, T. W. What is the Young's modulus of silicon? J. Microelectromechanical Syst. 19, 229-238, https://doi.org/10.1109/JMEMS.2009.2039697 (2010).

5. Fruhauf, J. Shape and Functional Elements of the Bulk Silicon Microtechnique (Springer-Verlag,Berlin/Heidelberg, 2005).

6. McAllister, D. V., Allen, M. G. \& Prausnitz, M. R. Microfabricated microneedles for gene and drug delivery. Annu. Rev. Biomed. Eng. 2, 289-313, https://doi.org/10.1146/annurev.bioeng.2.1.289 (2000).

7. Larrañeta, E., Lutton, R. E., Woolfson, A. D. \& Donnelly, R. F. Microneedle arrays as transdermal and intradermal drug delivery systems: Materials science, manufacture and commercial development. Mater. Sci. Eng. R: Reports 104, 1-32, https://doi. org/10.1016/j.mser.2016.03.001 (2016).

8. Reed, M. \& Lye, W.-K. Microsystems for Drug and Gene Delivery. Proc. IEEE 92, 56-75, https://doi.org/10.1109/JPROC.2003.820542 (2004).

9. Wilke, N. \& Morrissey, A. Silicon microneedle formation using modified mask designs based on convex corner undercut. J. Micromechanics Microengineering 17, 238-244, https://doi.org/10.1088/0960-1317/17/2/008ISTEX (2007).

10. Jansen, H., Gardeniers, H., de Boer, M., Elwenspoek, M. \& Fluitman, J. A survey on the reactive ion etching of silicon in microtechnology. J. Micromechanics Microengineering 6, 14-28, https://doi.org/10.1088/0960-1317/6/1/002ISTEX (1996).

11. Wind, R. A. \& Hines, M. A. Macroscopic etch anisotropies and microscopic reaction mechanisms: a micromachined structure for the rapid assay of etchant anisotropy. Surf. Sci. 460, 21-38, https://doi.org/10.1016/S0039-6028(00)00479-9ISTEX (2000).

12. Belougne, J. \& Caillard, C. Method and Apparatus for Etching a Substrate (2018).WO2018127561.

13. Dierking, K. et al. Unusual regulation of a STAT protein by an SLC6 family transporter in C. elegans epidermal innate immunity. Cell Host and Microbe 9, 425-435, https://doi.org/10.1016/j.chom.2011.04.011 (2011).

14. Stiernagle, T Maintenance of C. elegans, vol. https://doi.org/10.1895/wormbook.1.101.1 of WormBook (The C. elegans Research Community ed, http://www.wormbook.org 2006).

15. Pujol, N. et al. Anti-fungal innate immunity in C. elegans is enhanced by evolutionary diversification of antimicrobial peptides Plos Pathogens, 4, https://doi.org/10.1371/journal.ppat.1000105 (2008).

16. Ward, J. D. et al. Defects in the C. elegans acyl-CoA synthase, acs-3, and nuclear hormone receptor, $n h r-25$, cause sensitivity to distinct, but overlapping stresses Plos One, 9, https://doi.org/10.1371/journal.pone.0092552 (2014).

17. Zhu, Z. \& Lui, C Anisotropic Crystalline Etching Simulation (ACES). University of Illinois (1998).

18. Seidel, H., Csepregi, L., Heuberger, A. \& Baumgartel, H. Anisotropic Etching of Crystalline Silicon in Alkaline Solutions. J. Electrochem. Soc. 137, 15, https://doi.org/10.1149/1.2086277 (1990).

19. Offereins, H., Kühl, K. \& Sandmaier, H. Methods for the fabrication of convex corners in anisotropic etching of (100) silicon in aqueous KOH. Sensors Actuators A: Phys. 25, 9-13, https://doi.org/10.1016/0924-4247(90)87002-ZISTEX (1990)

20. Tang, B., Sato, K. \& Gosálvez, M. A. Sharp silicon tips with different aspect ratios in wet etching/drie and surfactant-modified tmah etching Sensors and Actuators A: Physical, 188, 220-229, https://doi.org/10.1016/j.sna.2012.01.031 (2012). Selected papers from The 16th International Conference on Solid-State Sensors, Actuators and Microsystems.

21. Wilke, N., Reed, M. L. \& Morrissey, A. The evolution from convex corner undercut towards microneedle formation: theory and experimental verification. J. Micromechanics Microengineering 16, 808-814, https://doi.org/10.1088/0960-1317/16/4/018ISTEX (2006).

22. Loer, C. M. et al. Cuticle integrity and biogenic amine synthesis in Caenorhabditis elegans require the cofactor tetrahydrobiopterin (BH4). Genetics 200, 237-53, https://doi.org/10.1534/genetics.114.174110 (2015).

23. Partridge, F. A., Tearle, A. W., Gravato-Nobre, M. J., Schafer, W. R. \& Hodgkin, J. The C. elegans glycosyltransferase bus-8 has two distinct and essential roles in epidermal morphogenesis. Dev Biol 317, 549-59, https://doi.org/10.1016/j.ydbio.2008.02.060 (2008). 
24. Liu, J.-H., Betzner, T. M. \& Henderson, H. T. Etching of self-sharpening (338) tips in (100) silicon. J. Micromechanics Microengineering 5, 18-24, https://doi.org/10.1088/0960-1317/5/1/004ISTEX (1995).

25. Schroder, H., Obermeier, E., Horn, A. \& Wachutka, G. K. M. Convex corner undercutting of 100 silicon in anisotropic KOH etching: the new step-flow model of 3-d structuring and first simulation results. J. Microelectromechanical Syst. 10, 88-97, https://doi. org $/ 10.1109 / 84.911096(2001)$.

26. Shikida, M. et al. A model explaining mask-corner undercut phenomena in anisotropic silicon etching: a saddle point in the etchingrate diagram Sensors and Actuators A: Physical, 97-98, 758-763, https://doi.org/10.1016/S0924-4247(02)00017-1 (2002). Selected papers from Eurosenors XV.

27. Gosálvez, M. A., Sato, K., Foster, A. S., Nieminen, R. M. \& Tanaka, H. An atomistic introduction to anisotropic etching. J. Micromechanics Microengineering 17, S1-S26, https://doi.org/10.1088/0960-1317/17/4/S01ISTEX (2007).

28. Han, J., Lu, S., Li, Q., Li, X. \& Wang, J. Anisotropic wet etching silicon tips of small opening angle in $\mathrm{KOH}$ solution with the additions of I2/KI. Sensors and Actuators A: Physical 152, 75-79, https://doi.org/10.1016/j.sna.2009.03.008 (2009).

29. Tanaka, H., Takeda, M. \& Sato, K. Si (100) and (110) etching properties in 5, 15,30 and $48 \mathrm{wt} \% \mathrm{KOH}$ aqueous solution containing Triton-X-100. Microsystem Technologies 23, 5343-5350, https://doi.org/10.1007/s00542-017-3368-y (2017).

30. Chisholm, A. D. \& Xu, S. The Caenorhabditis elegans epidermis as a model skin. ii: differentiation and physiological roles Wiley Interdiscip Rev. Dev Biol 1, 879-902, https://doi.org/10.1002/wdev.77ISTEX (2012).

31. Taffoni, C. et al. Microtubule plus-end dynamics link wound repair to the innate immune response. eLife 9 , https://doi.org/10.7554/ eLife.45047 (2020).

\section{Acknowledgements}

Supported by institutional grants from the Institut national de la santé et de la recherche médicale, Centre national de la recherche scientifique and Aix-Marseille University to the CIML, and the Agence Nationale de la Recherche program grants (ANR-12-BSV3-0001-01, ANR-16-CE15-0001-01, ANR-11-LABX-0054 (Labex INFORM) and ANR-11-IDEX-0001-02 (A*MIDEX)) to JJE. This work was partly supported by the French RENATECH network. Microfabrication and electron microscopy were performed in PLANETE CT PACA clean room facility. Worm sorting was performed using the facilities of the French National Functional Genomics platform, supported by the GIS IBiSA and Labex INFORM. The authors thank Shizue Omi and Roxane Fabre for help with worm optical imaging. Regarding the electron microscopy analyses, the authors thank the PiCSLFBI core electron microscopy facility (Aïcha Aouane, Fabrice Richard and Nicolas Brouilly, Aix Marseille Univ, CNRS, IBDM, Marseille, France), member of the national infrastructure France-BioImaging supported by the French National Research Agency (ANR-10-INBS-0004).

\section{Author contributions}

J.B., I.O. and J.J.E. conceived the experiments; J.B., C.C., I.O. and F.B. conducted pin array microfabrication; F.B. and I.O. the SEM observations; J.B., C.C. and J.J.E. the worm experiments; I.O. and J.J.E wrote the manuscript; All authors contributed to the discussion and reviewed the manuscript.

\section{Competing interests}

The authors declare no competing financial interests.

\section{Additional information}

Supplementary information is available for this paper at https://doi.org/10.1038/s41598-020-60333-7.

Correspondence and requests for materials should be addressed to I.O. or J.J.E.

Reprints and permissions information is available at www.nature.com/reprints.

Publisher's note Springer Nature remains neutral with regard to jurisdictional claims in published maps and institutional affiliations.

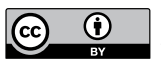

Open Access This article is licensed under a Creative Commons Attribution 4.0 International License, which permits use, sharing, adaptation, distribution and reproduction in any medium or format, as long as you give appropriate credit to the original author(s) and the source, provide a link to the Creative Commons license, and indicate if changes were made. The images or other third party material in this article are included in the article's Creative Commons license, unless indicated otherwise in a credit line to the material. If material is not included in the article's Creative Commons license and your intended use is not permitted by statutory regulation or exceeds the permitted use, you will need to obtain permission directly from the copyright holder. To view a copy of this license, visit http://creativecommons.org/licenses/by/4.0/.

(C) The Author(s) 2020 